\author{
Military Technical \\ College \\ Kobry El-Kobba \\ Cairo, Egypt
}

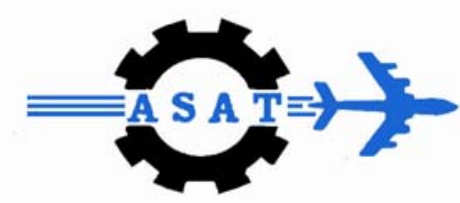

12-th International

Conference on

Aerospace Sciences \&

Aviation Technology

\title{
PERFORMANCE OF NON LINEAR ADAPTIVE INTERFERENCE CANCELLER IN DS/CDMA SYSTEMS
}

\author{
Khairy El Barbary ${ }^{1}$
}

\begin{abstract}
There are several advantages to spread the spectrum of the signal, which you wish to transmit, over the channel. The major advantages are summarized as follows: The ability to asynchronous multiple access without need for flow control, mitigation of the multipath propagation delay and the inherent ability of spread spectrum to reject interfering signals whose bandwidths are small compared to that of the spread spectrum signal. However, narrow band jamming sources could degrade the performance of the DS/CDMA single user detector by introducing extremely low signal to interference ratio $(S I R)$ at the input of the single user matched filter. This paper provides an adaptive interference canceller (AIC) which effectively reduces the narrow band interference and consequently improves the single user detection capabilities.
\end{abstract}

\section{KEY WORDS}

Adaptive Interference Canceller (AIC)

Non- Linear Adaptive Filter (NAF) 


\section{I- INTRODUCTION}

In direct sequence code division multiple access (DS/CDMA) communication systems, each user transmits symbols modulated upon a unique spreading sequence [1]. The conventional matched correlator demodulates the transmitted symbols of a specific user by correlating the received signal with a synchronized replica of the spreading waveform of interest. The optimal detection performance can be achieved when all the spreading waveforms are orthogonal or only one user is active [2]. However, the orthogonal property can not be easily achieved, owing to random timing offsets between different users in an asynchronous system. A nearby interfering user of large power will deteriorate the reception of the highly attenuated signal. In addition co-channel narrow band interference may be powerful enough so that communications becomes effectively impossible. Thus the matched correlator is vulnerable to the near-far problem, as well as co-channel interference signals. Consequently, the system capacity is limited by the multiple access interference (MAI) and narrow band interference reduction capabilities [3].

Many interference suppression schemes, previously introduced [4,5], can mitigate this near-far problem by exploring the structure of the MAl. These schemes are significantly more complex than the matched filter receiver and require explicit knowledge or estimates of interference parameters. Interference suppression schemes based on the minimum mean-squared error (MMSE) criterion, was motivated after the MMSE equalizers show ability to suppress both intersymbol interference (ISI) and cross talk interference in wire channels. In order to improve the performance of spread spectrum communication link in an interference corrupted environments without increasing the transmit bandwidth (or reducing throughput) some additional means of interference reduction must be used. Also in order to handle the increasing number of subscribers, it will be necessary to develop improved signal recovery algorithms that can operate in severe co-channel signal environments.

The narrow band interference (NBI) rejection capability of DS/CDMA systems can be further enhanced by employing interference rejection filters prior to signal despreading [6]. The objective of the adaptive filter is to obtain a high-quality estimate of a desired signal in the presence of co-channel interference signals. Usually linear adaptive filter,(LAF) is implemented as a transversal filter with finite impulse response (FIR) adopted by either the direct matrix inversion method, the least mean square (LMS) method or the recursive least squares (RLS). These filters show ability to reduce the effect of narrow band interference on expense of some throughput reduction due to utilizing a training sequence.

It has been demonstrated that nonlinear adaptive filter, perform significantly better than those linear ones $[7,8]$. This paper verifies the performance of the non linear adaptive filters $[6,9]$ as an interference rejection filters in DS/CDMA systems. The second part of this paper introduces a non-linear adaptive filter followed by a memory less non-linear limiter for reducing the effect of narrow-band interference [8]. The behavior of these systems is evaluated, through computer simulations, for the case of narrow-band binary phase shift keying (NB-BPSK) interference signals for the DS/CDMA system. The results are represented in terms of the improvement factor of the signal-to-noise ratio (SNR) and the matched filter output for the corrected signal by the considered system. Comments on the obtained results and their significance are introduced in the last section. 


\section{II- Description of DS/CDMA single user Detector with interference canceller}

The use of adaptive filter for CDMA applications, such as mobile communication systems is of interest because of the potential for higher channel capacity and extended range. An overview of the concepts and improved performance promised by adaptive filters, along with an extensive bibliography of relevant research, could be found in [8]. A block diagram of the DS-CDMA single user detector with interference canceller is shown in Fig.1.The received signal $x(t)$ is assumed to be consisting of the desired user signal, $s(t)$, the narrow band interference, $i(t)$ and an additive background noise, $n(t)$. The received signal $x(t)$ has the form

$$
x(t)=s(t)+n(t)+i(t)
$$

Where $n(t)$ is a white, Gaussian noise with zero-mean and variance $\sigma_{n}^{2}$. It represents an approximation of both the multiple access interference, MAI, and multipath interference, MPI in addition to the receiver thermal noise. The spread spectrum signal transmitted by a single user is given by, [2]

$$
s(t)=d(t) p(t) \cos \omega_{0} t
$$

Where, $d(t)$ represents rectangular data symbols, $( \pm 1)$ with a duration, $T_{b}$ seconds. $P(t)$ represents binary un-correlated pseudo random-noise $(P N)$ code $( \pm 1)$ with chip duration, Tc seconds. Usually the ratio $\left(T_{b} / T_{c}\right)=N$ is the length of the spreading code which represents the processing gain of the spread spectrum system, and $\omega_{0}$ is the carrier frequency. The narrow band interference (NBI), is assumed to be represented in the general form, as

$$
i(t)=A m(t) \cos \left(\left(\omega_{0}+\Omega\right) t+\phi\right)
$$

Where A and $\phi$ are the amplitude and phase of the NBI respectively and $\Omega$ is the carrier offset of the interference signal from the carrier of the desired spread spectrum signal. The model defined by equation (3), is referred as the narrow-band model, since it is assumed that the interference signal is narrow-band relative to the carrier frequency. The desired spread spectrum signal also assumed to be temporally uncorrelated with the NBI signal and with the background noise.

The interference modulating signal $m(t)$ is assumed to be digitally modulated interference where $m(t)= \pm 1$ with a duration of $T_{j}$ seconds, $T_{j} \gg T_{b}$. That the bandwidth of the interference signal is much narrower than that of the desired spread spectrum signal.

It is assumed that the integrator used in the down conversion in Fig.1 is an ideal one, which will not distort the desired signal or the NBI. The sampling rate is assumed equal to the chip rate $\left(1 / T_{c}\right)$. Thus, the input of the interference canceller is given by

$$
z_{k}=s_{k}+n_{k}+i_{k}
$$

Where, the low pass signals $s_{k}$ and $i_{k}$ are given by

$$
\begin{gathered}
s_{k}=d(k) p(k) \\
i_{k}=A m(k) \cos \left(\Omega T_{c} k\right)
\end{gathered}
$$

Before leaving the description of the problem, it is convenient to introduce the limitation on the performance of the single user detector. Usually the SIR is very low due to existence of narrow band signal in the same frequency band of the desired spread spectrum signal. The output of a matched filter, which is shifted version of the code auto correlation function, will be completely distorted by the narrow band 
interference consequently, this leads to large probability of decision errors. Thus, it is required to enhance the SIR at the input of the de-spreading filter in order to obtain good detection performance. It is also worth to note that the model of the received signal in equations (1) approximates both the effect of multipath interference and other user's interferences. This approximation is acceptable due to the assumption of existence of relatively high power NBI accompanying the multiple access signals. However, an optimum de-spreading filter is capable to reduce the effect of these two sources of interference.

The next coming section introduce different algorithms for controlling non-linear adaptive filter (NAF) as an interference canceller. The results are introduced, for each algorithm, in terms of the corrected correlation function and the improvement factor, which is considered as the ratio of the SIR at the output of the interference canceller to that one at its input.

\section{III-- Structure of the non-linear adaptive interference canceller (AIC)}

The non-linear interference canceller (NIC) structure is shown in Fig.3 [11,12]. It consists of a finite impulse response (FIR), transversal filter of order M. The output of this $\operatorname{FIR}\left(\hat{z}_{k}\right)$ represents an estimate for the coming sample, $Z_{\mathrm{k}}$. The error between the estimated value and the actual one $\left(\varepsilon_{k}\right)$ is computed and it is utilized as the argument of the nonlinear function $\rho($.) which is defined as

$$
\begin{aligned}
& \qquad \rho\left(\varepsilon_{k}\right)=\varepsilon_{k}-\tanh \left(\frac{\varepsilon_{k}}{\sigma_{k}^{2}}\right) \\
& \text { Where, } \sigma_{k}^{2} \text { is defined as } \sigma_{k}^{2}=\Delta_{k}-1
\end{aligned}
$$

$\Delta_{k}$ is the sample estimate of the prediction error variance which is calculated as

$$
\Delta_{k}=\beta \Delta_{k-1}+(1-\beta) \varepsilon_{k}^{2}
$$

Where, $\beta$ is the forgetting factor with value between zero and one. The memory-less limiter is used to overcome the impulsive distortion caused by phase change of the interference signal. Its function is given by [13]

$$
g(x)=\frac{2}{h}\left[\frac{1}{2}-Q\left(\frac{x}{c}\right)\right]
$$

Where $Q($.$) is the standard error function of the normal curve. The parameter c$ control the limiting effect and $h$ is a scaling parameter. It worth to note that if $g(x)=x$; we have the ordinary non-linear adaptive filter (NAF). The low-pass equivalent of the received signal is a composite signal of three components and it is explained by equation (5) in section II. At the time instant k, we define the following M-dimensional vectors.

$$
\begin{gathered}
X_{k}=\left[Z_{k-1} Z_{k-2} \ldots \ldots . Z_{k-M}\right]^{T} \\
\widetilde{X}_{k}=\left[\widetilde{Z}_{k-1} \widetilde{Z}_{k-2} \ldots \ldots . \widetilde{Z}_{k-M}\right]^{T} \\
W_{k}=\left[w_{1, k} w_{2, k} \ldots \ldots \ldots w_{M, k}\right]^{T}
\end{gathered}
$$

where $X_{k}, \widetilde{X}_{K}$ and $\boldsymbol{W}_{\mathrm{k}}$ are the observation vector, the non-linear filter output and the weights vector of the FIR filter respectively. The adaptive algorithm for the non-linear adaptive filter is given by [13-14]

$$
W_{k}=W_{k-1}+\frac{\mu}{\gamma_{k}} \rho\left(\varepsilon_{k}\right) \widetilde{X}_{k}
$$


where $\gamma_{k}$ is an estimate for the input power to the filter at time instant $\mathrm{k}$, defined as

$$
\left.\gamma_{k}=\gamma_{k-1}+\left.\mu|| X_{k}\right|^{2}-\gamma_{k-1}\right\rfloor
$$

The considered performance measure of this non-linear interference canceller is the improvement factor. It is defined as the ratio of the signal-to-interference ratio at the input of the canceller $(S I R)_{0}$ to that one at its input. Thus the improvement factor for the signal canceller $\left(I M F_{A I C}\right)$ is given by [11]:

$$
I M F_{A I C}=10 \log \left[E\left(\left|z_{k}-s_{k}\right|^{2}\right) / E\left(\left|Y_{k}-s_{k}\right|^{2}\right)\right]
$$

where the input signal-to-interference ratio is defined as

$$
S N R_{I}=10 \log \left[E\left(\left|s_{k}\right|^{2}\right) / E\left(\left|Z_{k}-s_{k}\right|^{2}\right)\right]
$$

While the improvement factor in case of non linear adaptive filter (IMF $\left.F_{N A F}\right)$ is defined as [10]

$$
I M F_{N A F}=\frac{S I R_{0}}{S I R_{i}}=\frac{E\left\{\left[z_{k}-S_{k}\right]^{2}\right\}}{E\left\{\left[\hat{z}_{k}-S_{k}\right]^{2}\right\}}
$$

\section{IV- SIMULATION OF BEHAVIOR OF AIC AND NAF FOR REJECTION OF NARROW-BAND INTERFERENCE}

The narrow band interference is represented as narrow band, binary phase shift keying (NBPSK) signal with symbol duration, $T_{j}$. Thus, the low pass equivalent of this signal is given by

$$
i_{k}=A_{k} \cos \left(\Omega T_{C} k\right)
$$

where $A_{k}= \pm 1$ and $\Omega=\pi / 10$; is the offset frequency of the interference signal from the carrier of the desired DS/CDMA signal. The constructed simulation is actuated upon the distorted signal in equation (5) and the results are represented in table-1. The results show that, the obtained IMF versus the bandwidth ratio of the desired signal, $B_{c}$ (Binary phase shift keying data signal modulating a maximum length sequence of length 127 chips), to that one of the interference signal, $B_{j}$. Table-2 shows the IMF versus the input SIR. The results indicate that the narrower the interference bandwidth the more reduction of the effect of the interference by the non-linear canceller. Also as the interference, power increases the improvement factor increases. The interference canceller, consisting of adaptive non-linear filter followed by a limiter provides much improvement than the non-linear adaptive filter.

Table-1 Effect of Bandwidth ratio on the Improvement factor.

\begin{tabular}{|l|l|l|l|l|l|}
\hline$B_{c} / B_{j}$ & 100 & 200 & 300 & 400 & 500 \\
\hline$I M F_{A I C}$ & 25.3163 & 29.3947 & 29.5434 & 29.1619 & 29.5448 \\
\hline$I M F_{N A F}$ & 12.9682 & 15.5241 & 19.3196 & 17.9784 & 18.0386 \\
\hline
\end{tabular}

Table-2 Effect of input SIR on the Improvement factor.

\begin{tabular}{|l|l|l|l|l|l|}
\hline$S I R(d B)$ & -20 & -15 & -10 & -5 & 0.0 \\
\hline$I M F_{A I C}$ & 30.5316 & 27.4150 & 24.1466 & 19.7741 & 14.8568 \\
\hline$I M F_{N A F}$ & 16.3332 & 15.9674 & 14.9526 & 12.6600 & 9.1224 \\
\hline
\end{tabular}

The output of the correlator (matched filter for the desired spreading signal) is presented in Fig.3. Two cases were considered for the input signal, on the first we assume that the input to the matched filter is only a desired signal, the corresponding output is shown in Fig.3(a). While the second case, the input is the distorted signal 
as in equation (5). The input SIR was assumed to be $-20 \mathrm{~dB}$, and the output of the matched filter for the distorted input is presented in Fig.3 (b). One can see that the narrow-band binary phase shift keying (NB-PSK) interference signal is completely hidden the desired signal.

Fig.4. illustrates the convergence behavior of the non-linear algorithm. In Fig.5 the output of the matched filter for the corrected signal is plotted, along with that one for the desired signal. It is clear that the inserted interference canceller removed the NBBPSK interference signal while it maintains the desired one.

\section{V- CONCLUSIONS}

The matched filter (or the correlator receiver) is an optimum detection scheme for DSICDMA signals in presence of additive white Gaussian noise. Three common problems limits the capacity of DSICDMA systems. Namely, the near- far problem, MAI \& MPI and Narrow band interference. The first two problems are usually overcomed by controlling the despreading filter in such away to reduce their effect while mainting an acceptable detection performance. On the other hand reducing the effect of NB interference requires insertion of interference canceller in front of the despreading filter. Two scheme for NB interference cancellation are introduced, namely, NAF and AIC. Both of the NAF and AIC shows ability to reduce the NB interference effect while in comparison the AIC outperforms the NAF in NB cancellation on the expense of adding a memory-less limiter to overcome the impulsive distortion caused by phase change of the interference signal.

\section{REFERENCES}

[1] Prasad, R 'CDMA for Wireless Personal Communications' Artech House, Boston London, 1996.

[2] Proakis, J.G. 'Digital Communications' McGRaw-Hill, New York, 1995, (3rd edn).

[3] L.M. Li and L.B. Milstein "Rejection of NB interference in PN spread spectrum systems using transversal filters," IEEE Trans Comm., Vol. Com-30, pp.925-928, May 1982.

[4] U.Madhow and M. L. Honig "MMSE interference suppression for DS/SS CDMA," IEEE Trans Comm., Vol. Com-42 No. 12, pp.3178-3188, Dec.1994.

[5] R. Lupas and S. Verdu, "Near-far resistance of multiuser detectors for asynchronous channels," IEEE.J. Selected Areas Comm., vol.38 ,pp 496-508, April 1990

[6] C.Carlemalm, H.V. Poor and A. Logothetis, "Suppression of Multiple Narrow Band Interference in a Spread Spectrum Communication system," IEEE.J. Selected Areas Comm., vol.18, No. 8, pp 1365-1373, Aug. 2000.

[7] L.A. Rusch and H.V. Poor "Narrow band interference suppression in CDMA spread spectrum communications," IEEE Trans Comm, Vol. Com-42, pp.1969-1979, April 1994.

[8] Y. Li and Z. Ding. "Convergence analysis of finite length blind adaptive equalizers." IEEE Trans. Signal Processing, vol. 43. pp. 2120-2129, Sept. 1995.

[9] E. Masry, "Closed form analytical results for the rejection of narrow band interference in PN spread spectrum systems - part-I : Linear prediction filters, " IEEE Trans Comm, Vol. Com-32, pp.888-896, Aug. 1984.

[10] Haykin, S. 'Adaptive filter theory ' Prentice - Hall, N.J, 1991 (2 ${ }^{\text {nd }}$ edn).

[11] R. Vijayan and H.V. Poor, "Non Linear techniques for interference suppression in SS systems," IEEE Trans Comm., Vol. Com-38, pp.1060-1065, July 1990.

[12] L. Garth and H.V. Poor "Narrow- band interference suppression in impulsive channel," IEEE Trans Aerosp. Electro. Sys., Vol. AES 28, pp.15-34, Jan 1992. 
[13] K.J. Wang and Y.Yao "New non-linear algorithms for narrow-band interference suppression in CDMA spread spectrum systems, "IEEE journal on selected area in Comm, Vol. Com-17 No. 12, pp.2148-2151, Dec 1999.

[14] W. R. Wu" New non linear algorithms for Estimating and suppressing narrow-band interference in DS spread spectrum systems," IEEE Trans Comm, Vol. Com-44, pp.508-515, April 1996.

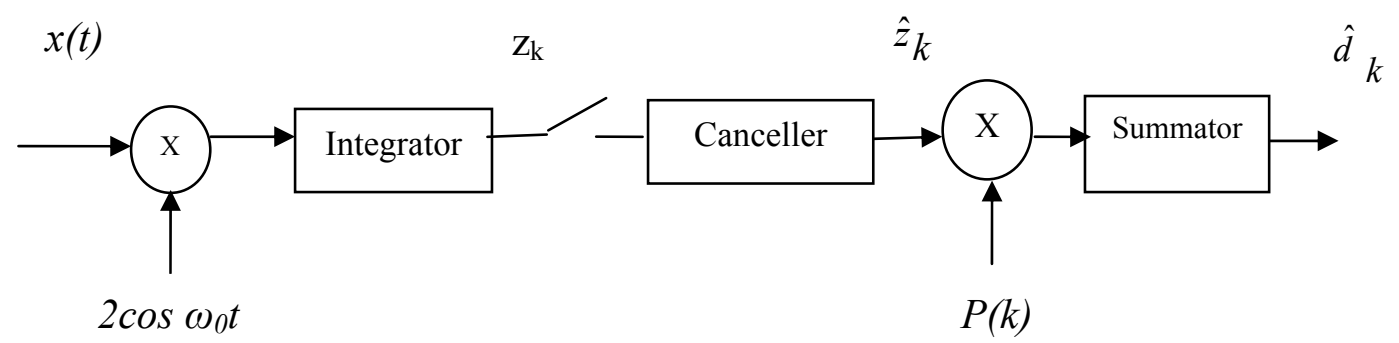

Fig.1. DS/CDMA single user detector with interference canceller.

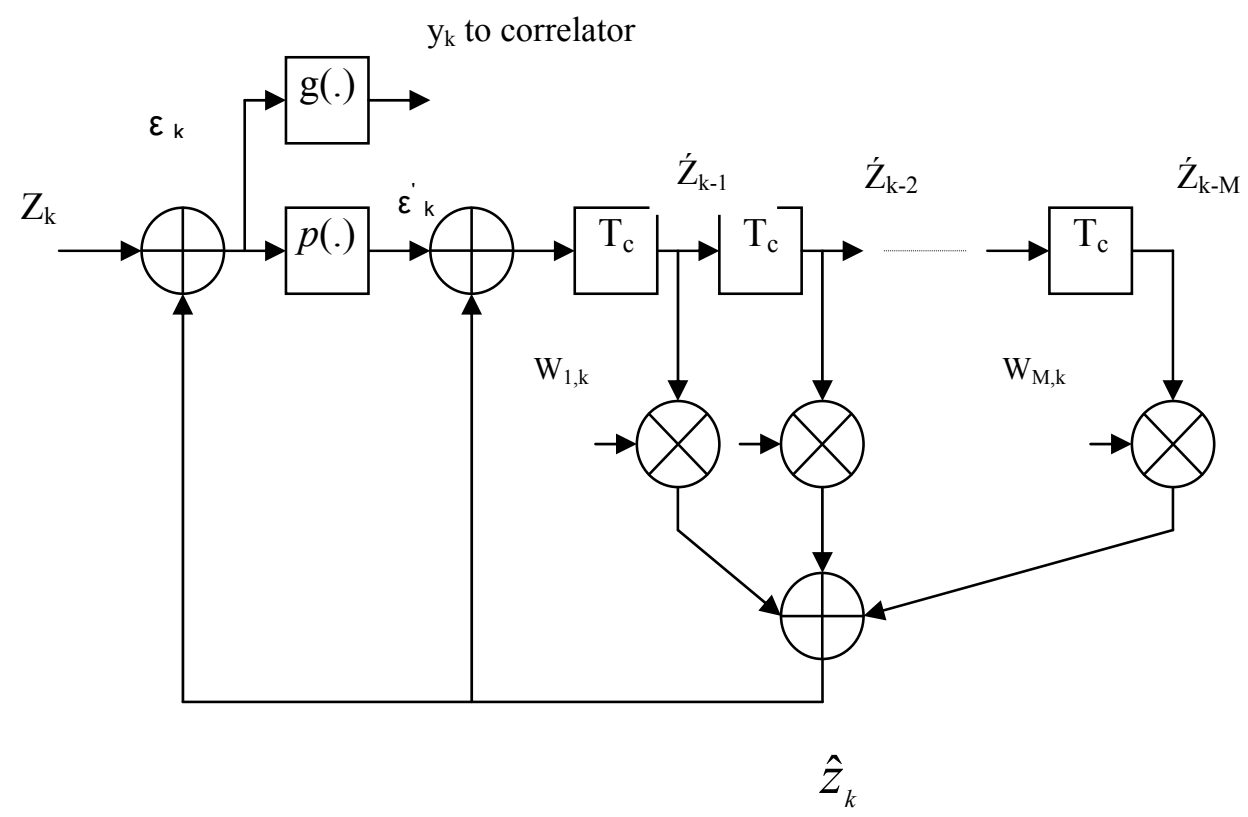

Fig2. Non-linear adaptive interference canceller with limiter. 
(a) MF output for pure signal

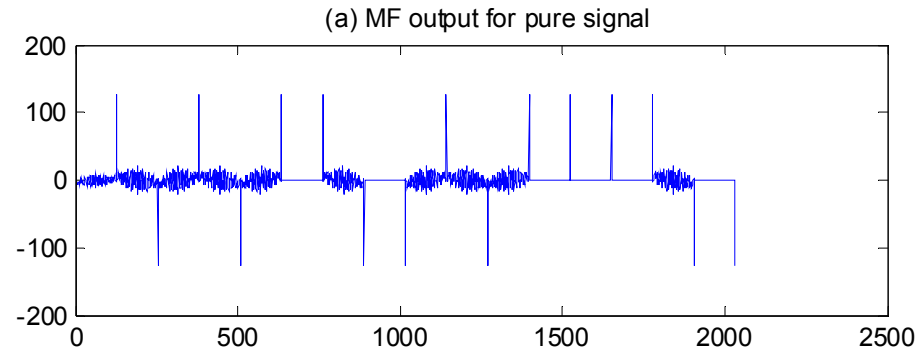

(b) MF output for corrupred signal

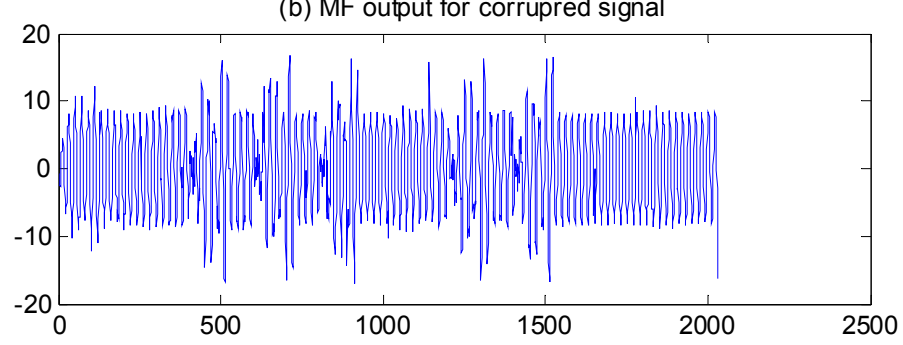

Fig.3 The effect of NB-BPSK interference on MF output.

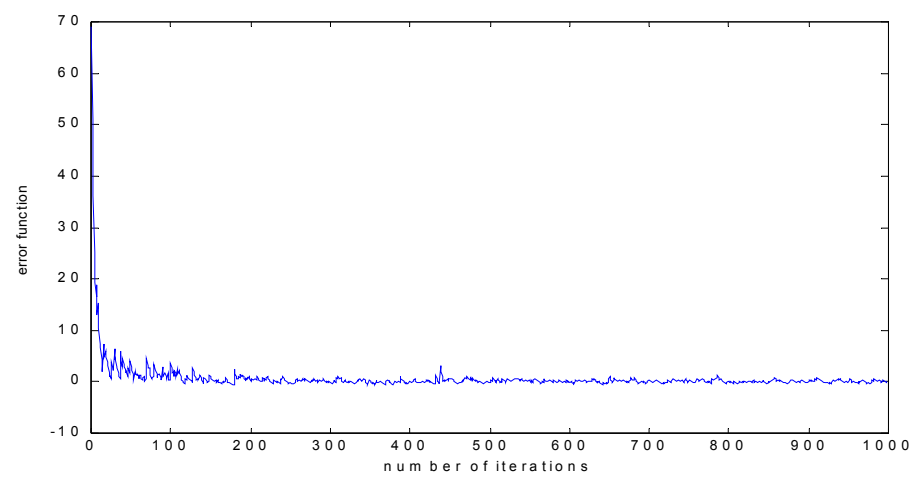

Fig.4 Convergence of the error function of the interference canceller.

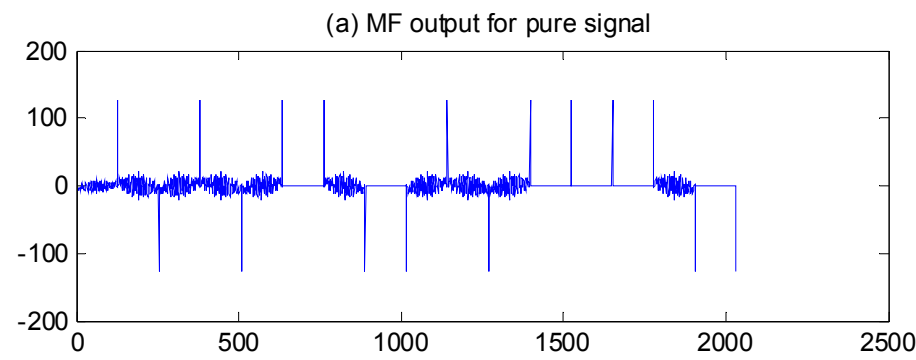

(b) MF output for corrected signal

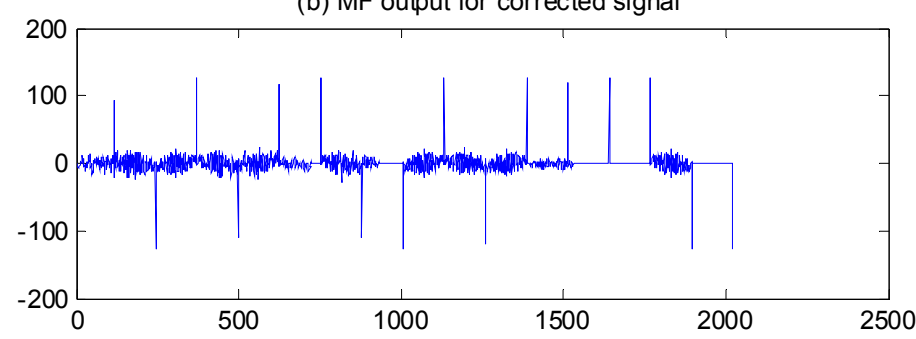

Fig.5. The output of the MF for the corrected signal. 